\title{
Subsalt illumination studies through longitudinal and transversal wave propagation
}

Riaz Alai *, Jan Thorbecke and Eric Verschuur, Delft University of Technology, The Netherlands

Copyright 2007, SBGf - Sociedade Brasileira de Geofísica

This paper was prepared for presentation at the $10^{\text {th }}$ International Congress of The Brazilian Geophysical Society held in Rio de Janeiro, Brazil, 19-22 November 2007.

Contents of this paper were reviewed by the Technical Committee of the $10^{\text {th }}$ International Congress of the Brazilian Geophysical Society and do not necessarily represent any position of the SBGf, its officers or members. Electronic reproduction, or storage of any part of this paper for commercial purposes without the written consen of the Brazilian Geophysical Society is prohibited.

\begin{abstract}
In this paper, propagation and illumination studies have been performed for acoustic and elastic wave field propagation through a realistic model representing a typical Gulf of Mexico salt structure. Strong wave conversions may occur at the interfaces of sediments and adjacent salt bodies. These wave conversions occur because of the high velocity contrasts and inhomogeneities, and in general (longitudinal) P-waves get scattered, refracted and converted due the stronger wave distortion effects of (longitudinal) $\mathrm{P}$-waves at these strong velocity contrasts, yielding large shadow zones. On the other hand, the converted (transversal) S-waves, propagate in general in the salt bodies with less wave front distortion because of their lower propagation velocity and, therefore smaller contrasts with the surrounding sediment $P$-wave velocities.
\end{abstract}

\section{Introduction}

In general, when processing offshore data, the top-salt events are picked and a P-wave salt flood migration is performed to determine the base-salt structure. The remaining subsurface velocity model is being estimated in a step-wise approach. To emphasize the importance of Swave propagation inside salt bodies, some experiments have been performed on a realistic model representing a typical Gulf of Mexico salt structure : the Ziggy model, shown in Figure 1.

The Sub-salt Multiples Attenuation and Reduction Technology Joint Venture (SMAART JV) released, to the industry and universities, the 3-D numerical model referred to as Ziggy. The Ziggy geologic model represents a generalized structure of exploration interest found along the Sigsbee Escarpment in the deep water Gulf of Mexico. The 3-D physical Ziggy model (see Figure 1) is a layered model with a complex salt structure, with dimensions of $38 \times 42 \times 10 \mathrm{~km}$ depth (see also van Veldhuizen and Blacquière, 2005).

Marine-type data was recorded over the 3-D Ziggy model, by placing the model in the physical modeling facility at the Delft University of Technology (Blacquière and Volker, 2002).
Figure 2 shows a picture of the Delft Experimental Facility for Imaging. Specific subsurface scale models have been designed and placed in this water tank, for recording data over realistic physical models.

In seismic data imaging, the subsurface model is not known and due to wave conversions it often becomes difficult to identify the base of salt and sub-salt structures. Lu et al. (2003) outlined the identification, removal and imaging of P-S conversions at salt-sediment interfaces and showed the importance of taking wave conversions into account in migration. These situations typically occur when recording offshore data over a subsurface containing salt bodies (e.g. in the Gulf of Mexico).

As mentioned earlier, recording offshore data over a subsurface with salt bodies, strong wave conversions may occur at the top and base of the salt body. A large amount of this (longitudinal) $\mathrm{P}$-wave energy gets converted into (transversal) S-waves and these S-waves show up in the seismic data as echoes of the P-wave reflections. (Alai and Verschuur, 2006) outlined that with the aid of physical model data, these effects can be studied in a controlled manner. Experiments on a physical model dataset illustrate clearly that wave conversions appear at salt body interfaces and that migration of this data for a known "subsurface model" with the correct subsurface velocities (either $\mathrm{P}$ or $\mathrm{S}$ ) will provide the correct images of (multiply) mode-converted energy.

Subsurface illumination studies are very important in determining shadow zones in seismic data. Xie et al. (2006) discussed a one-way wave-equation based method for seismic illumination analysis by extrapolating the wave fields from sources and receivers to the subsurface target.

\section{Acoustic and elastic wave field propagation}

Figure 3a shows a 2-D vertical section from the 3-D Ziggy velocity depth model. As can be observed there is thinshaped salt body present in this section. The salt body as used in this section contains velocities for (longitudinal) Pwave propagation. To illustrate the importance of (transversal) S-wave propagation within salt bodies, the following experiment has been performed. The thinshaped salt body has been replaced with a lower velocity being the (transversal) S-wave velocity. Figure $3 b$ shows the 2-D vertical section from the Ziggy model, the upper salt body $\mathrm{P}$-wave velocity has been replaced by $\mathrm{S}$-wave velocity.

\section{Examples}

Using both 2-D sections from Figure 3, data has been modeled for (acoustic) $P$ and (elastic) $S$ wave field 
propagation through the thin salt body. Figure 4 shows various modeled snapshots for acoustic and elastic wave field propagation. In Figure 4a P-wave snapshots are shown for 3 source locations along the surface. From these snapshots it can be noticed, that the wave fronts of the propagating P-waves get distorted, as the wave is propagating through high velocity contrasts between sediments and salt body. Note that in these examples only transmission effects (downward one-way wave field propagation) are shown and reflection effects are not considered here.

However, the propagating wave front through the salt is the wave front that illuminates the subsalt structures. The more complex this wave front is, the more difficult it will be to image these subsalt structures. As the wave field hits the top of the salt body, some distortion effect is clearly visible (see arrow in Figure 4a). This effect, causing a triplication in the wave front, is quite strong, and is directly related to the strength of the velocity contrast between the sediments and the top of the salt body. A similar experiment has also been performed for the model depicted in Figure 3b. In this model the P-wave salt body has been replaced by a lower (transversal) S-wave velocity. Because the velocity contrast is less than the previous experiment (with P-wave salt body), we expect less distortion effects, and overall delayed subsalt images because of the slower wave field propagation inside the salt body.

Figure $4 \mathrm{~b}$ shows the S-wave snapshots for 3 source locations at the surface (note here the delayed waves because of slower S-wave velocity propagation velocity through the subsurface model). From these snapshots comparisons, it can be concluded that for larger velocity contrasts, the distortion effect of the wave front is larger. Figure $4 \mathrm{c}$ shows the combined $\mathrm{P}$ - and $\mathrm{S}$-wave snapshots - the blue colored waves represent $P$-waves and the red colored waves represent S-waves.

Figure $4 \mathrm{~d}$ is also a combination of $\mathrm{P}$-wave and S-wave snapshots but for another source location at the surface. In Figure 4d it also observed that the S-waves have a higher illumination angle than the P-waves.

Note that because of the fast velocity behavior of $\mathrm{P}$ waves, the wave front gets distorted and becomes more complex and locally focused.

On the other hand less wave front distortion effects are observed for propagating S-waves, due to the fact that the S-wave velocity within the salt matches more the Pwave velocities of the surrounding sediments.

For better illustration of the wave field propagation, some illuminating beams are calculated and illustrated in Figure 5. The illuminating beams are shown here for P-wave and S-wave propagation through the thin salt body. Here the wave field is extrapolated from the surface into the subsurface and per depth level, the energy of the total wave field has being calculated. Figure $5 a$ shows the $P$ wave beams for 3 source locations along the surface.

Figure $5 \mathrm{~b}$ shows the S-wave beams for 3 source locations at the surface. Note that S-waves get not distorted and scattered as much as P-waves: the effect is that S-waves propagate with higher illumination angle within salt bodies and therefore may better illuminate the base-salt and subsalt structures. Figure $5 \mathrm{c}$ shows the addition of a series of illuminating beams with sources along the surface for only P-waves.

Figure $5 d$ shows the addition of a series of illuminating beams with sources along the surface for only S-wave propagation (in the salt body). Notice that P-wave propagation creates more shadow zones, and note again that the S-wave propagation better illuminates the basesalt and subsalt structures.

\section{Conclusions}

From the illumination studies carries out on a realistic model representing a typical Gulf of Mexico salt structure, it can be concluded that illumination of the base-salt reflector and the subsalt region is better obtained by the wave field that have a wave-conversion at the salt body boundaries, i.e. that propagate with the P-wave velocity in the sediments and with the S-wave velocity within the salt. Because the S-wave velocity within the salt matches very well the $\mathrm{P}$-wave velocities of the surrounding sediments, less distortion during the propagation is observed and the illumination of the subsalt region is more uniform. On the other hand, the full P-wave propagation creates strong inhomogeneities in the illumination of the subsalt structures, yielding large shadow zones.

\section{Acknowledgments}

The authors would like to thank the SMAART JV consortium for providing the ZIGGY model and the sponsoring companies of the DELPHI consortium for their financial support.

\section{References}

Alá'i, R, and Verschuur, D.J., 2006, Subsalt imaging of wave converted reflections in physical model data, $68^{\text {th }}$ EAGE Conference \& Exhibition.

Blacquière, G. and Volker, A.W.F., 2002, Physical Modelling and Subsalt Illumination, $64^{\text {th }}$ Mtg.: Eur. Assn. Geosci. Eng., C044.

Lu, R.S., Willen D.E., and Watson, I.A., 2003, Identifying, removing and imaging P-S conversions at salt-sediment interfaces, Geophysics, 68, 1052-1059.

van Veldhuizen, E.J. and Blacquière, G., 2005, Integrated approach to acquisition geometry analysis, 75th Ann. Internat. Mtg.: Soc. of Expl. Geophys., ACQ1.1. Xie., X., Jin, S. and Wu, R., 2006, Wave-equation based seismic illumination analysis, Geophysics, 71, S169-S177 

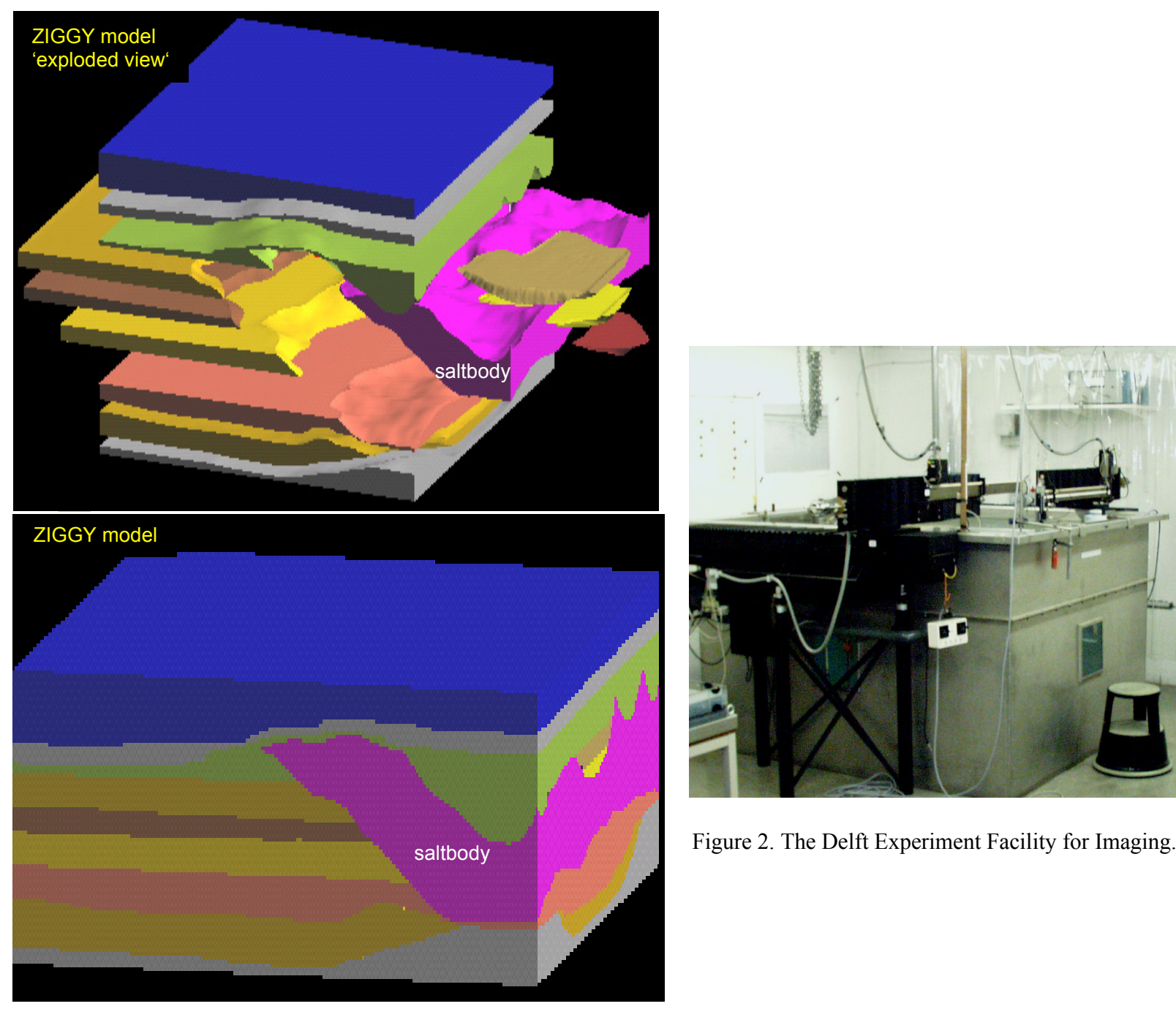

Figure 2. The Delft Experiment Facility for Imaging.

Figure 1. The 3-D Ziggy model.
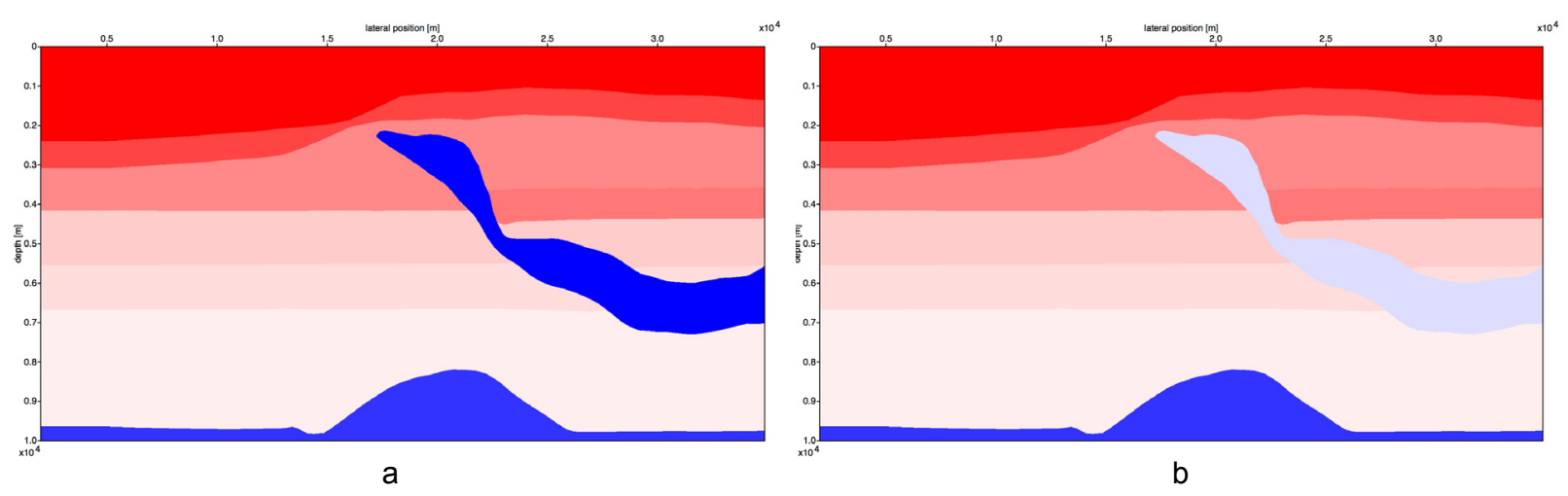

Figure 3. a) 2-D vertical section from the Ziggy model. b) 2-D vertical section from the Ziggy model, the upper salt body $\mathrm{P}$-wave velocity has been replaced by $\mathrm{S}$-wave velocity. 

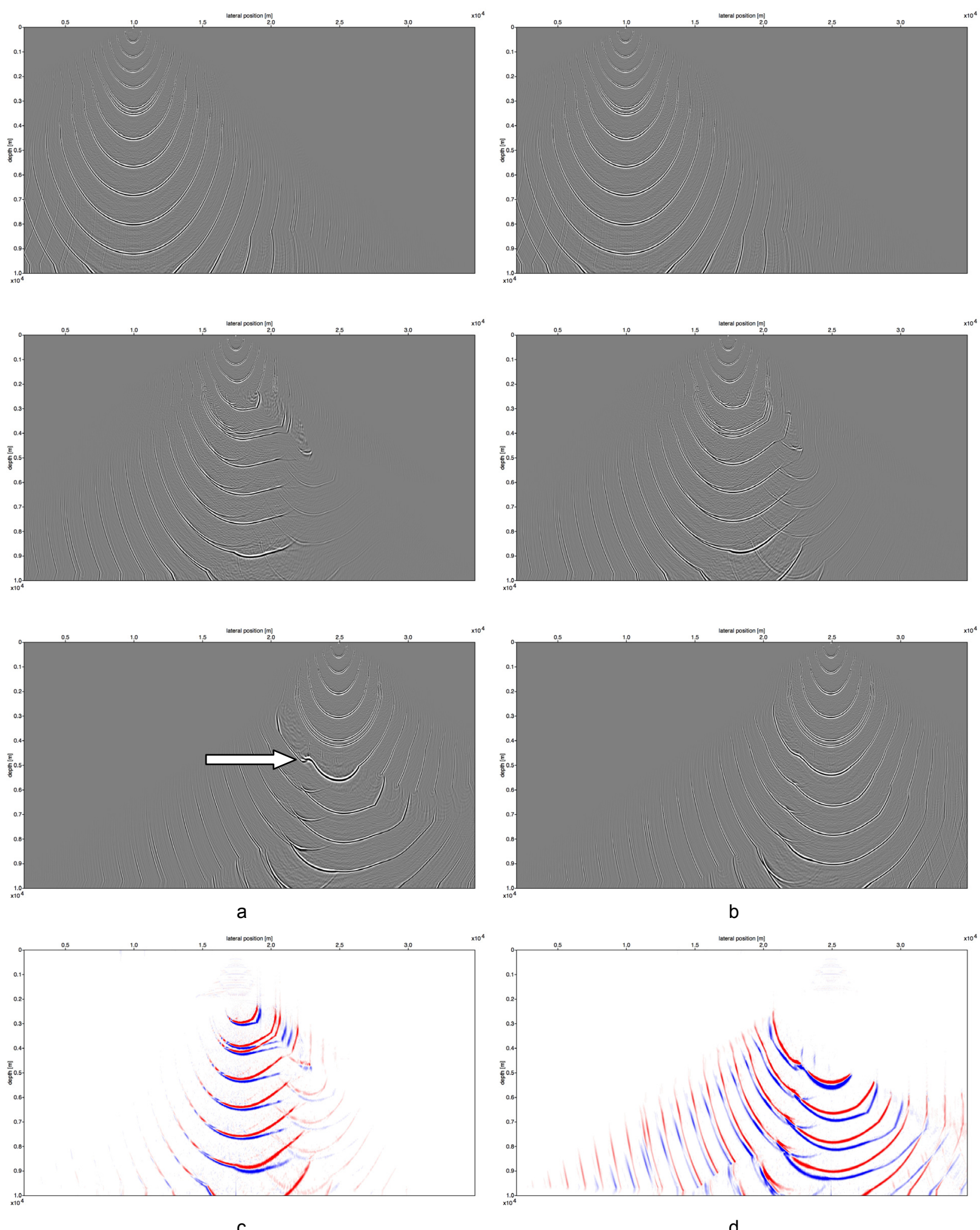

Figure 4. Various modeled snapshots for acoustic and elastic wave field propagation : a) P-wave snapshots for 3 source locations along the surface. b) S-wave snapshots for 3 source locations along the surface (note here the delayed waves because of slower $\mathrm{S}$-wave propagation velocity through the subsurface model). c) Combined P- and S-wave snapshots - the blue colored waves represent $\mathrm{P}$-waves and the red colored waves represent S-waves. d) Same as c), but for another source location at the surface. Note that because of the fast velocity behavior of P-waves, the wave front gets distorted and gets more complex and focused. On the other hand for S-waves the wave front distortion effects are less observed (propagation with higher illumination angle). 

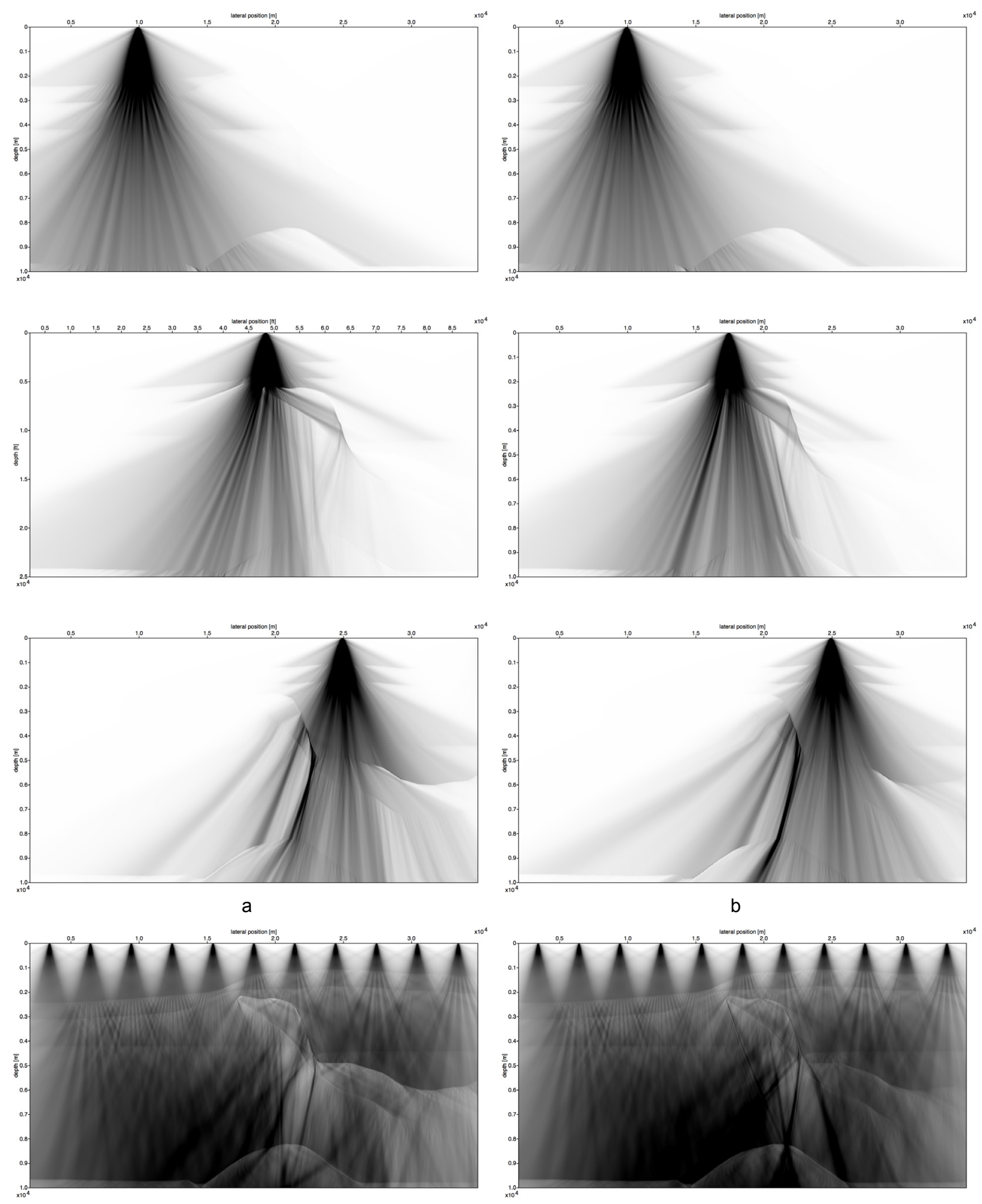

C

d

Figure 5. Illuminating beams showing the wave propagation through the subsurface model : a) P-wave beams for 3 source locations along the surface. b) S-wave beams for 3 source locations along the surface. Note that S-waves do not get distorted and scattered as much as P-waves. Therefore, the S-waves may be better suited to illuminate the base-salt and subsalt structures. c) Addition of a series of illuminating beams with sources along the surface for only P-waves. d) Addition of a series of illuminating beams with sources along the surface for only S-waves (in the salt body). Notice that P-wave propagation creates more shadow zones, and note again that the S-wave propagation illuminates better the base-salt and subsalt structures. 